\title{
TWO CASES WITH PRIMARY THYROID LYMPHOMA DEVELOPED ON THE BASIS OF HASHIMATO'S THYROIDITIS
}

\author{
Hande Peynirci ${ }^{1}$, Pinar Sisman ${ }^{1}$, Cigdem Aksu ${ }^{2}$, Ozen Oz Gul ${ }^{1}$, \\ Soner Cander ${ }^{3}$, Erdinc Erturk ${ }^{1}$, Canan Ersoy ${ }^{1}$
}

\author{
${ }^{1}$ Department of Internal Medicine, Division of Endocrinology and Metabolism, Uludag University Faculty of \\ Medicine, Bursa; Turkey \\ ${ }^{2}$ Department of Internal Medicine, Uludag University Faculty of Medicine, Bursa; Turkey \\ ${ }^{3}$ Sevket Y1lmaz Hospital, Bursa; Turkey
}

\section{Objectives:}

\section{Cases:}

\section{Discussion}

Primary thyroid lymphomas are rare cancers and account for $\% 1-5$ of all thyroid cancers and $\% 3$ of all extranodal NHLs. Development of thyroid lymphoma is sixty-times more common among cases with Hashimato's thyroiditis than cases without thyroiditis. It was hypothesized that association between Hashimato's thyroiditis and primary thyroid lymphoma is due to chronic lymphocytic stimulation as promoting factor to malignant transformation. Therefore, we aimed to present cases of thyroid lymphoma encountered in our clinic.

\section{CASE 1:}

A 54-year old female patient admitted with pain and swallow on the cervical region. In ultrasonographic(USG) thyroid examination, it was found that both thyroid lobes were very large with routh parenchyme consistent with chronic thyroiditis; also there was a solid nodule $23 \mathrm{~mm}$ in diameter in left side. In fine needle aspiration biopsy(FNAB) of thyroid, there were suspicious findings strongly indicating lymphoid hyperplasy. Patient underwent total thyroidectomy. Pathology results were consistent with extranodal (MALT type) marginal zone lymphoma. There were no pathologic findings implicating systemic involvement; patient is still followed-up.

CASE 2:

A 40-year old female patient admitted with hypothyroidism. In USG examination of thyroid, it was found that thyroid parenchyme was extensively heterogeneous and there were multiple nodules. FNAB findings were reported as Hashimato's thyroiditis and papillary carcinoma. Patient underwent total thyroidectomy and pathology results were considered as morphologic and immunephenotypic findings consistent with extranodal (MALT type) marginal zone lymphoma. Patient underwent radiotherapy. She is in remission and still followed-up.

Primary thyroid lymphomas are rare cancers and commonly seen in women aged 50-80 years. Cases usually admit with growing mass in cervical region and it can be accompanied with coarsed voice, disphagy, dyspnea and stridor. Its only known risk factor is Hashimato's thyroiditis and Hashimato's thyroiditis has been found in \%94 of the primary thyroid lymphoma cases. Primary thyroid lymphomas majorly have B-cell origin and there are other histologic types consistent with MALT lymphoma, diffuse B-cell lymphoma (DLBCL) or both of them. Subtype and stage of lymphoma are important for prognosis and treatment choice. MALT lymphoma of thyroid has best prognosis with $\% 100$ of 5-year survival expentancy. Chemotherapy regiments, especially rituximab; are main treatment approach in MALT lymphoma cases together with DLBCL and DLBCL whereas combined chemotherapy and radiotherapy are main treatment approach in bulky disease.

In conclusion, total thyroidectomy or radiotherapy are curative only in localized MALT lymphoma. In this regard, early diagnosis is important. 